\title{
Dynamic Control Applied to a Laboratory Antilock Braking System
}

\author{
Cuauhtémoc Acosta Lúa, ${ }^{1}$ Bernardino Castillo Toledo, ${ }^{2}$ Stefano Di Gennaro, ${ }^{3}$ \\ and Marcela Martinez-Gardea ${ }^{1}$ \\ ${ }^{1}$ Departamento de Ciencias Tecnológicas, Universidad de Guadalajara, Centro Universitario de la Ciénega \\ Avenida Universidad 1115, 47820 Ocotlán, JAL, Mexico \\ ${ }^{2}$ Centro de Investigación y de Estudios Avanzados (CINVESTAV) del IPN, Unidad Guadalajara, Avenida Científica, \\ Colonia El Bajío, 4010 Zapopan, JAL, Mexico \\ ${ }^{3}$ Department of Information Engineering, Computer Science and Mathematics, Center of Excellence DEWS, \\ University of L'Aquila, Via G. Gronchi 18, 67100 L’Aquila, Italy
}

Correspondence should be addressed to Cuauhtémoc Acosta Lúa; cuauhtemoc.acosta@cuci.udg.mx

Received 11 September 2014; Revised 30 December 2014; Accepted 30 December 2014

Academic Editor: Mehmet Onder Efe

Copyright (C) 2015 Cuauhtémoc Acosta Lúa et al. This is an open access article distributed under the Creative Commons Attribution License, which permits unrestricted use, distribution, and reproduction in any medium, provided the original work is properly cited.

\begin{abstract}
The control of an antilock braking system is a difficult problem due to the existence of nonlinear dynamics and uncertainties of its characteristics. To overcome these issues, in this work, a dynamic nonlinear controller is proposed, based on a nonlinear observer. To evaluate its performance, this controller has been implemented on an ABS Laboratory setup, representing a quarter car model. The nonlinear observer reconstructs some of the state variables of the setup, assumed not measurable, to establish a fair benchmark for an ABS system of a real automobile. The dynamic controller ensures exponential convergence of the state estimation, as well as robustness with respect to parameter variations.
\end{abstract}

\section{Introduction}

The antilock braking system (ABS) was developed to prevent the wheels from locking up while braking. This prevents the slippage of the wheels on the surface, adjusting the brake fluid pressure level of each wheel, and helps the driver to keep the control on the vehicle [1-3]. In fact, the ABS is designed to increase the braking efficiency, maintaining the manoeuvrability of the vehicle and reducing the driving instability, while decreasing the braking distance. Modern ABS systems try to not only prevent the wheels from locking up, but also aim to obtain maximum wheel grip on the surface while the vehicle is braking $[4,5]$. The technical difficulties in successfully implementing the antilock concept contained in the 1936 patent for an "apparatus for preventing lock braking of wheels in a motor vehicle," by Robert Bosch [6], were solved between 1967 and 1970, when Mercedes-Benz engineers changed the mechanical sensors for contactless sensors operating under the induction principle [7]. Finally, when the electronic integrated circuits were small and robust enough, it was possible to record data from the wheel's sensors and to use more reliable actuators for imposing brake hydraulic pressure. The mass production started with the ABS second generation, in 1978 [7]. With the hardware technology breakthroughs, now the challenge is to propose efficient control algorithms for the actuators. Several algorithms had been aimed for controlling the ABS; see $[8,9]$ for interesting overviews.

In this paper, a mechatronic system, the ABS Laboratory setup, manufactured by Inteco Ltd., is used to implement new control strategies and to compare them, avoiding the high costs of tests on real full-sized vehicles. The setup represents a quarter car model and consists of two rolling wheels. The lower wheel, made of aluminum, imitates the relative road motion of the car, whereas the upper wheel, made of rigid plastic, is mounted to the balance lever and simulates the wheel of the vehicle. In order to accelerate the lower wheel, a large DC motor is coupled to it. The upper wheel is equipped with a disk-brake system, driven by a small DC motor [10]. Earlier works on this kind of setup are mainly based on 
the assumption that information of all sensors is available for measurement. Some articles discuss the use of controllers with comparative (linear and nonlinear) control techniques as in [11-13]. In [14-18], control strategies based on slidingmode analysis are analyzed. An optimal controller of an ABS Laboratory setup is presented in [19]. Finally, other works deal with fuzzy controllers $[20,21]$ or other intelligent control techniques such as adaptive neurofuzzy $[22,23]$ and neurofuzzy techniques [24].

While these previous works are based on the assumption that the angular velocities of the two setup wheels are available for measuring, in this paper we consider that only the upper wheel angular velocity is measurable. In fact, it corresponds to the angular velocity of the automobile wheel, while the angular velocity of the lower setup wheel corresponds to the longitudinal velocity of a real vehicle, which is rarely measured and has to be estimated. Therefore, an observer is proposed and the well-known Lyapunov technique is used [25] to ensure the exponential convergence. The (constant) observer gains are determined via an offline numerical procedure. With the same Lyapunov technique, a dynamic controller is designed to impose the exponential convergence of the slip to a desired reference.

Previous results on observer design for this kind of setup can be found in the literature. For instance, in [26] an observer-based direct adaptive fuzzy neural controller is proposed. A nonlinear observer of the vehicle longitudinal velocity is given in [27]. In [28], an observer for the velocity estimation is introduced, in presence of varying friction and road bank angles. Other types of linear and nonlinear observers, based on sliding-mode technique, can be found in $[29,30]$. In [30], an extended kalman filter is proposed, using a real-time solution of a Riccati differential equation.

With respect to the previous results on observers and controllers, the proposed dynamic controller has an advantage in its simplicity, attractive for real-time implementation on the ECU. To better show its performance, we have also compared the proposed dynamic controller with the slidingmode one proposed in [16], showing better performances in terms of input smoothness, implying less noise and better wear resistance, and also in terms of braking distance.

The paper is organized as follows. Section 2 briefly presents the mathematical model of the experimental setup. Section 3 is dedicated to the design of the dynamic controller. The results are discussed in Section 4, where a comparison between the controller proposed in this paper and a slidingmode control is performed. Finally, some conclusions are presented.

\section{Mathematical Model of the Experimental ABS Laboratory Setup}

In this paper, an $A B S$ Laboratory setup manufactured by Inteco Ltd. has been studied; see Figure 1. It represents a quarter car model and consists of two rolling wheels. The lower aluminum wheel emulates the road motion, whereas the upper plastic wheel simulates the vehicle wheel. In order to accelerate the lower wheel, a DC motor is coupled to it,

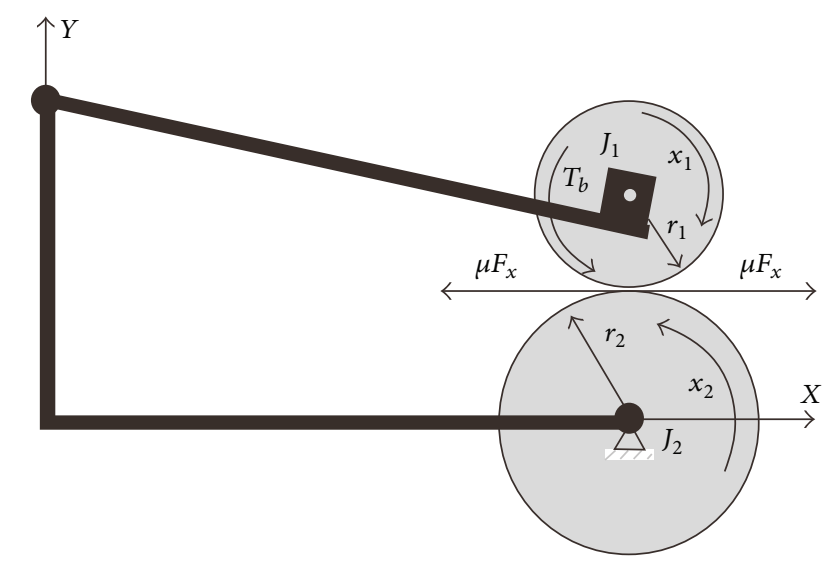

FIGURE 1: Scheme of the ABS Laboratory setup.

while the upper wheel is equipped with a disk-brake system. Encoders on the wheels allow determining the positions and velocities, through differential quotients, of the two wheels. Although simple, this setup preserves the fundamental characteristics of an actual ABS system in the range $0-70 \mathrm{~km} / \mathrm{h}$ [10].

The control problem is to impose a desired wheel slip, that is, to set at a desired value the relative difference of the contact point velocities between the two wheels. On actual vehicles, the longitudinal velocity is estimated applying a relationship between the wheel angular velocity and its radius, considering the wheels deformation very small compared to its dimensions. This estimation is good, as long as no slip is present. Unfortunately, since the braking process involves a slip between the wheel and the road surface, when it occurs it is not possible to estimate the vehicle longitudinal velocity in this way. In this case, an observer is necessary to estimate the longitudinal velocity.

The mathematical model of the ABS Laboratory setup is derived under the assumptions of negligible lateral and vertical motions and rolling resistance force negligible with respect to braking (see Figure 2). The braking torque, $T_{b}$, and the bearing friction torque, $M_{10}$, act on the upper wheel. The bearing friction torque, $M_{20}$, acts on the lower wheel. The tractive force, $F_{x}$, acts on both wheels. The dynamic equations of the ABS Laboratory setup are [10]

$$
\begin{gathered}
\dot{x}_{1}=\frac{r_{1}}{J_{1}} F_{x} s-\frac{1}{J_{1}}\left(d_{1} x_{1}+M_{10}+T_{b}\right) s_{1}, \\
\dot{x}_{2}=-\frac{r_{2}}{J_{2}} F_{x} s-\frac{1}{J_{2}}\left(d_{2} x_{2}+M_{20}\right) s_{2},
\end{gathered}
$$

where $x_{1}, x_{2}$ are the angular velocities of the upper and lower wheels, whose inertia moments are $J_{1}, J_{2}$ and whose radii are $r_{1}, r_{2}$. Furthermore, $d_{1}, d_{2}$ are the viscous friction coefficients of the upper and lower wheels (the nominal parameters are given in Table 1$)$ and $s(x), s_{1}\left(x_{1}\right)$, and $s_{2}\left(x_{2}\right)$ are auxiliary variables

$$
\begin{gathered}
s(x)=\operatorname{sign}\left(r_{2} x_{2}-r_{1} x_{1}\right), \\
s_{1}\left(x_{1}\right)=\operatorname{sign}\left(x_{1}\right), \quad s_{2}\left(x_{2}\right)=\operatorname{sign}\left(x_{2}\right),
\end{gathered}
$$




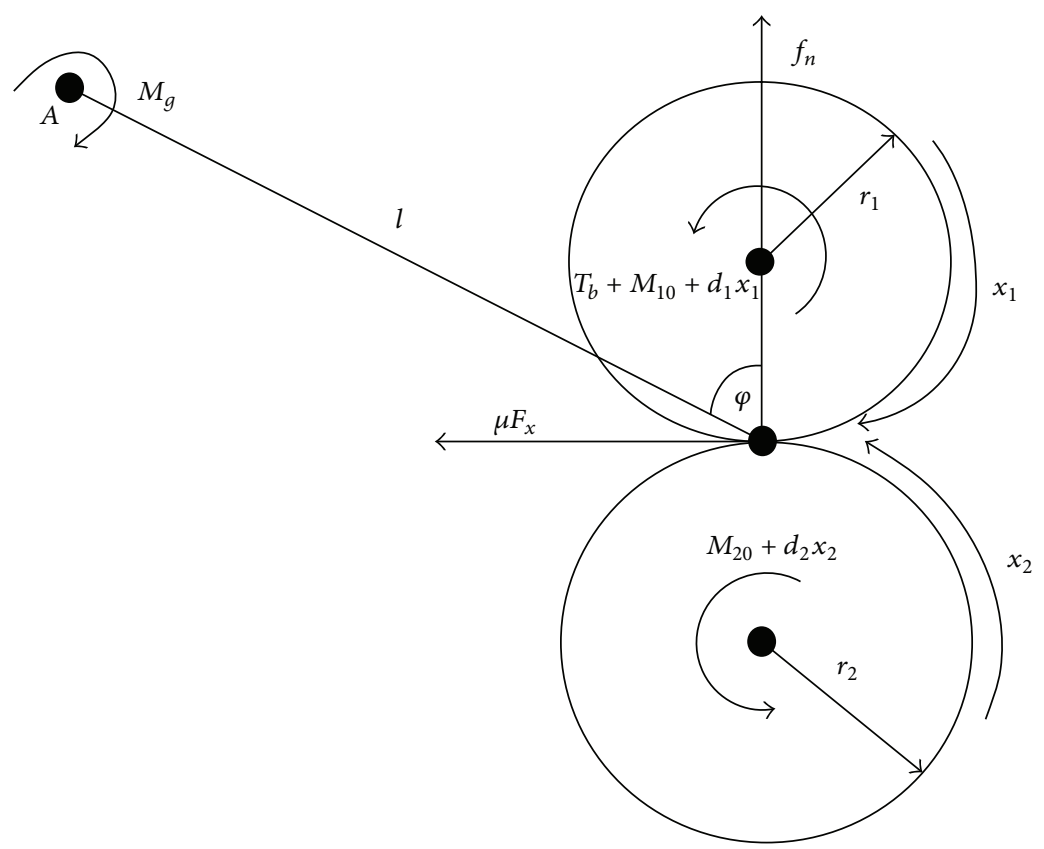

FIGURE 2: Forces and torques acting on the ABS Laboratory setup.

used to determinate if the vehicle is in the traction mode or in the braking mode, with

$$
\operatorname{sign}(x)= \begin{cases}1, & \text { if } x>0 \\ 0, & \text { if } x=0 \\ -1, & \text { if } x<0\end{cases}
$$

Here, $r_{1} x_{1}$ represents the vehicle wheel velocity, while $r_{2} x_{2}$ represents the vehicle velocity. Additionally, $F_{x}$ represents the tractive force generated at the contact between upper wheel and lower wheel. Various models are available in the literature to model the tire behavior [31]. Without loss of generality, since the same approach can be used with different tire models [27], in this work Pacejka's "magic formula" was chosen to describe this force. This formula approximates the response curve of the braking process based on experiments test data. It is widely used and allows us to work with a wider range of values, including the linear and nonlinear regions of the tire characteristic:

$$
F_{x}=\mu_{x} D_{x} \sin \left(C_{x} \arctan \left(B_{x} \lambda\right)\right),
$$

where

$$
\lambda=\frac{r_{2} x_{2}-r_{1} x_{1}}{r_{2} x_{2}}
$$

is the wheel slip, that is, the relative difference of the wheel velocities. The force depends on positive experimental coefficients, given by the stiffness factor $B_{x}$, the shape factor $C_{x}$, and the peak value $D_{x}$, which are determined to match the experimental data. Moreover, $\mu \in[0,1]$ is the friction coefficient between the upper and lower wheels. Figure 3 shows the behavior of the tractive force calculated with (4) as a function of the wheel slip (5). Note that $F_{x}\left(\lambda_{2}\right)>F_{x}\left(\lambda_{1}\right)$ for $\lambda_{2}>\lambda_{1}$.

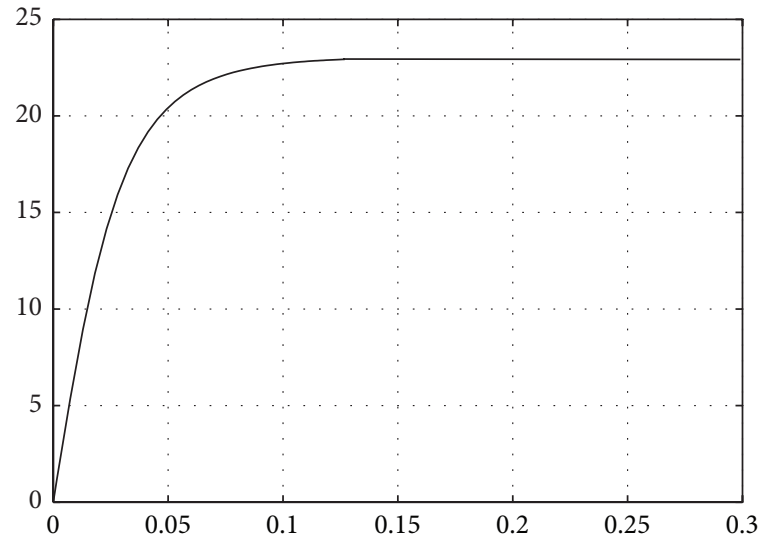

FIgure 3: The tractive force $F_{x}$ as a function of the wheel slip $\lambda(\mathrm{N}$ versus s).

The braking torque $T_{b}$ is modeled with a first-order equation [10], given by

$$
\dot{T}_{b}=-c_{31} T_{b}+c_{31} b(u),
$$

where $c_{31}$ is a positive constant and $b(u)$ describes the relation with the control input applied to the DC motor. This latter drives the action of the brake pads, with the control input $u \in$ $[0,1]$, and generates the braking torque $T_{b}$. This relation can be approximated by

$$
b(u)= \begin{cases}b_{1} u+b_{2}, & \text { if } u \geq u_{0}, \\ 0, & \text { if } u<u_{0}\end{cases}
$$

where $u_{0}$ is the operating threshold of the brake driving system. According to the mathematical model, (7) is similar to the brake pedal in an automobile $[6,11,14,16,18,21,32]$. 
TABLE 1: Coefficients and system variables, for the ABS Laboratory setup.

\begin{tabular}{|c|c|c|}
\hline$x_{1}$ & Angular velocity of the upper wheel & $\mathrm{rad} / \mathrm{s}$ \\
\hline$x_{2}$ & Angular velocity of the lower wheel & $\mathrm{rad} / \mathrm{s}$ \\
\hline$T_{b}$ & Braking torque & $\mathrm{Nm}$ \\
\hline$r_{1}$ & Radius of the upper wheel & $0.0995 \mathrm{~m}$ \\
\hline$r_{2}$ & Radius of the lower wheel & $0.0990 \mathrm{~m}$ \\
\hline$J_{1}$ & Upper wheel inertia moment & $7.54 \times 10^{-3} \mathrm{Kg} \mathrm{m}^{2}$ \\
\hline$J_{2}$ & Lower wheel inertia moment & $25.6 \times 10^{-3} \mathrm{Kg} \mathrm{m}^{2}$ \\
\hline$d_{1}$ & $\begin{array}{l}\text { Upper wheel viscous friction } \\
\text { coefficient }\end{array}$ & $118.74 \times 10^{-6} \mathrm{Kg} \mathrm{m}^{2} / \mathrm{s}$ \\
\hline$d_{2}$ & $\begin{array}{c}\text { Lower wheel viscous friction } \\
\text { coefficient }\end{array}$ & $214.68 \times 10^{-6} \mathrm{Kg} \mathrm{m}^{2} / \mathrm{s}$ \\
\hline$\mu$ & Friction coefficient between wheels & 1 \\
\hline$M_{10}$ & Static friction of the upper wheel & $0.0032 \mathrm{~N} \mathrm{~m}$ \\
\hline$M_{20}$ & Static friction of the lower wheel & $0.0925 \mathrm{~N} \mathrm{~m}$ \\
\hline$b_{1}$ & Constant & 15.24 \\
\hline$b_{2}$ & Constant & -6.21 \\
\hline$c_{31}$ & Constant & $20.37 \mathrm{~s}^{-1}$ \\
\hline$u_{0}$ & Constant & 0.415 \\
\hline$u$ & Control input & \\
\hline$D_{x}$ & Peak value & 23 \\
\hline$C_{x}$ & Shape factor & 1.68 \\
\hline$B_{x}$ & Stiffness factor & 28 \\
\hline$\gamma$ & Observer gain & 21 \\
\hline$k_{o 1}$ & Observer gain & 140 \\
\hline$k_{o 2}$ & Observer gain & 40 \\
\hline$k_{s 0}$ & Controller gain & 19 \\
\hline$k_{s 1}$ & Controller gain & 32 \\
\hline
\end{tabular}

Under normal operation conditions, the wheel velocity $r_{1} x_{1}$ matches the forward velocity $r_{2} x_{2}$. When the brake is applied, braking forces are generated at the wheel interface; $r_{1} x_{1}$ will tend to be lower than $r_{2} x_{2}$ (but always positive), and a slippage will occur. This braking process and the slip between the wheel and the road surface imply that it is not possible to consider the vehicle velocity as known, here represented by $r_{2} x_{2}$. Therefore, in the following section an observer, $\hat{x}_{2}$, will be designed during the braking phase, assuming that the state variable $x_{1}$ is measurable and the disturbances $M_{10}, M_{20}$ are known. During braking the wheel slip, $\lambda$, is positive, as well as $x_{1}, x_{2}$, so

$$
\begin{gathered}
s=\operatorname{sign}\left(r_{2} x_{2}-r_{1} x_{1}\right)=1, \quad s_{1}=\operatorname{sign}\left(x_{1}\right)=1, \\
s_{2}=\operatorname{sign}\left(x_{2}\right)=1,
\end{gathered}
$$

and (1) becomes

$$
\begin{gathered}
\dot{x}_{1}=\frac{r_{1}}{J_{1}} F_{x}-\frac{1}{J_{1}}\left(d_{1} x_{1}+M_{10}+T_{b}\right), \\
\dot{x}_{2}=-\frac{r_{2}}{J_{2}} F_{x}-\frac{1}{J_{2}}\left(d_{2} x_{2}+M_{20}\right) .
\end{gathered}
$$

\section{Design of a Dynamic Controller}

According to the mathematical model derived in (9), the following observer is proposed:

$$
\begin{gathered}
\dot{\bar{x}}_{1}=\frac{r_{1}}{J_{1}} \widehat{F}_{x}-\frac{1}{J_{1}}\left(d_{1} \widehat{x}_{1}+M_{10}+T_{b}\right)+k_{o 1}\left(x_{1}-\widehat{x}_{1}\right), \\
\dot{\hat{x}}_{2}=-\frac{r_{2}}{J_{2}} \widehat{F}_{x}-\frac{1}{J_{2}}\left(d_{2} \widehat{x}_{2}+M_{20}\right)+k_{o 2}\left(x_{1}-\widehat{x}_{1}\right),
\end{gathered}
$$

where $k_{o 1}, k_{o 2}>0$ are the observer gains designed hereinafter and

$$
\begin{array}{r}
\widehat{F}_{x}=F_{x}(\widehat{\lambda})=\mu_{x} D_{x} \sin \left(C_{x} \arctan \left(B_{x} \hat{\lambda}\right)\right), \\
\hat{\lambda}=\frac{r_{2} \widehat{x}_{2}-r_{1} \widehat{x}_{1}}{r_{2} \widehat{x}_{2}} .
\end{array}
$$

In (10), the upper wheel velocity $x_{1}$ is assumed to be measurable and used as feedback term. In modern vehicles, equipped with ABS systems, this is an acceptable assumption in practice. Moreover, the system parameters, and in particular the bearing friction torques $M_{10}, M_{20}$, are assumed to be known, since they can be accurately identified [10].

In the following, the selection of the gains $k_{o 1}, k_{o 2}$ to guarantee the convergence of the observer is discussed. Let us consider the estimation errors

$$
e_{1}=x_{1}-\widehat{x}_{1}, \quad e_{2}=x_{2}-\widehat{x}_{2}, \quad e=\left(\begin{array}{ll}
e_{1} & e_{2}
\end{array}\right)^{T}
$$

and the error dynamics

$$
\begin{aligned}
& \dot{e}_{1}=-\left(k_{o 1}+\frac{r_{1}}{J_{1}}\right) e_{1}+\frac{r_{1}}{J_{1}}\left(F_{x}-\widehat{F}_{x}\right), \\
& \dot{e}_{2}=-k_{o 2} e_{1}-\frac{d_{2}}{J_{2}} e_{2}-\frac{r_{2}}{J_{2}}\left(F_{x}-\widehat{F}_{x}\right),
\end{aligned}
$$

calculated from (9) and (13). Let us consider the following Lyapunov candidate function:

$$
V_{o}=\frac{1}{2}\left(e_{1}^{2}+\gamma e_{2}^{2}\right)
$$

with $\gamma>0$.

Deriving (14) along the error dynamics (13), one works out

$$
\begin{aligned}
\dot{V}_{o}= & -\left(k_{o 1}+\frac{d_{1}}{J_{1}}\right) e_{1}^{2}-\gamma \frac{d_{2}}{J_{2}} e_{2}^{2}-\gamma k_{o 2} e_{1} e_{2} \\
& +\left(\frac{r_{1}}{J_{1}} e_{1}-\gamma \frac{r_{2}}{J_{2}} e_{2}\right)\left(F_{x}-\widehat{F}_{x}\right),
\end{aligned}
$$

where the term $F_{x}-\widehat{F}_{x}$ is calculated using the Lagrange mean value theorem $[27,28]$

$$
F_{x}-\widehat{F}_{x}=\left.\frac{\partial F_{x}}{\partial x_{1}}\right|_{\bar{\lambda}} e_{1}+\left.\frac{\partial F_{x}}{\partial x_{2}}\right|_{\bar{\lambda}} e_{2}=-c_{1} e_{1}+c_{2} e_{2}
$$


where $\bar{\lambda}=1-r_{1} \bar{x}_{1} /\left(r_{2} \bar{x}_{2}\right)$, with $\bar{x}_{1}, \bar{x}_{2}$ being some points between $x_{1}$ and $\widehat{x}_{1}$ and between $x_{2}$ and $\widehat{x}_{2}$. Moreover,

$$
\begin{gathered}
\frac{\partial F_{x}}{\partial x_{1}}=-\frac{1}{x_{2}} \frac{\mu_{x} D_{x} C_{x} B_{x}}{1+B_{x}^{2} \lambda^{2}} \cos \alpha(\lambda) \leq 0, \\
\frac{\partial F_{x}}{\partial x_{2}}=\frac{r_{2}(1-\lambda)}{r_{1} x_{2}} \frac{\mu_{x} D_{x} C_{x} B_{x}}{1+B_{x}^{2} \lambda^{2}} \cos \alpha(\lambda) \geq 0,
\end{gathered}
$$

where $\alpha(\lambda)=C_{x} \arctan B_{x} \lambda$.

Substituting (16) into (15), the derivative of the Lyapunov candidate along the error dynamics takes the form

$$
\begin{aligned}
\dot{V}_{o}= & -\left(k_{o 1}+\frac{d_{1}}{J_{1}}+\frac{r_{1}}{J_{1}} c_{1}\right) e_{1}^{2}-\gamma\left(\frac{d_{2}}{J_{2}}+\frac{r_{2}}{J_{2}} c_{2}\right) e_{2}^{2} \\
& -\left(\gamma k_{o 2}-\frac{r_{1}}{J_{1}} c_{2}-\gamma \frac{r_{2}}{J_{2}} c_{1}\right) e_{1} e_{2} .
\end{aligned}
$$

Let us analyze how it is possible to determine the observer gains, making use of a numerical procedure to upper-bound (18). First, setting $k_{o 1}=k_{o 2}=0$ in (18), one gets a quadratic form

$$
\begin{aligned}
\left.\dot{V}\right|_{\substack{k_{o 1}=0 \\
k_{o 2}=0}} & \\
= & -\left(\begin{array}{l}
e_{1} \\
e_{2}
\end{array}\right)^{T}\left(\begin{array}{cc}
\frac{d_{1}}{J_{1}}+\frac{r_{1}}{J_{1}} c_{1} & \frac{1}{2}\left(-\frac{r_{1}}{J_{1}} c_{2}-\gamma \frac{r_{2}}{J_{2}} c_{1}\right) \\
\frac{1}{2}\left(-\frac{r_{1}}{J_{1}} c_{2}-\gamma \frac{r_{2}}{J_{2}} c_{1}\right) & \gamma\left(\frac{d_{2}}{J_{2}}+\frac{r_{2}}{J_{2}} c_{2}\right)
\end{array}\right) \\
& \cdot\left(\begin{array}{l}
e_{1} \\
e_{2}
\end{array}\right)
\end{aligned}
$$

which depends on $c_{1}, c_{2}$. According to the definition (16) of $c_{1}, c_{2}$, one feasible way is to numerically calculate $\max \{\dot{V}\}$, making varying $x_{2}$ and $\lambda$, which are the variables appearing in the expressions (17). In this way, some values $x_{2}^{\circ}, \lambda^{\circ}$ and, correspondingly, some values $c_{1}^{\circ}, c_{2}^{\circ}$ remain determined. Hence, one gets

$$
\begin{aligned}
\dot{V}_{o} \leq & -\left(k_{o 1}+\frac{d_{1}}{J_{1}}+\frac{r_{1}}{J_{1}} c_{1}^{\circ}\right) e_{1}^{2}-\gamma\left(\frac{d_{2}}{J_{2}}+\frac{r_{2}}{J_{2}} c_{2}^{\circ}\right) e_{2}^{2} \\
& -\left(\gamma k_{o 2}-\frac{r_{1}}{J_{1}} c_{2}^{\circ}-\gamma \frac{r_{2}}{J_{2}} c_{1}^{\circ}\right) e_{1} e_{2} .
\end{aligned}
$$

Finally, it is possible to select the gains so that the following conditions are fulfilled:

$$
k_{o 1}>0, \quad k_{o 2}=\frac{r_{1}}{\gamma J_{1}} c_{2}^{\circ}+\frac{r_{2}}{J_{2}} c_{1}^{\circ}>0 .
$$

Conditions (21) ensure that the Lyapunov function derivative is negative definite

$$
\begin{aligned}
\dot{V}_{o} & \leq-\left(k_{o 1}+\frac{d_{1}}{J_{1}}+\frac{r_{1}}{J_{1}} c_{1}^{0}\right) e_{1}^{2}-\gamma\left(\frac{d_{2}}{J_{2}}+\frac{r_{2}}{J_{2}} c_{2}^{0}\right) e_{2}^{2} \\
& \leq-\lambda\left\|\begin{array}{l}
e_{1} \\
e_{2}
\end{array}\right\|^{2},
\end{aligned}
$$

where $\lambda=\min \left\{\left(k_{o 1}+d_{1} / J_{1}+\left(r_{1} / J_{1}\right) c_{1}^{\circ}\right), \gamma\left(d_{2} / J_{2}+\left(r_{2} / J_{2}\right) c_{2}^{\circ}\right)\right\}>$ 0 . Hence, the error system (13) is exponentially stable to the origin; that is, the estimation errors (12) tend exponentially to zero, with a time constant at least $\tau=1 / \lambda$. This proves that the observer (10), with the gains (21), ensures the exponential convergence of the estimates $\widehat{x}_{1}, \widehat{x}_{2}$ to the states variables $x_{1}$, $x_{2}$, respectively.

The second part of this section is devoted to the design of the control law ensuring that $\lambda$ tends to the desired reference. To this aim, let us consider the slip error $\widehat{e}=\widehat{\lambda}-\lambda_{\text {ref }}$ and its dynamics, nonsingular for $\widehat{x}_{2} \neq 0$,

$$
\begin{aligned}
\dot{\hat{e}}= & -\frac{1}{\widehat{x}_{2}}\left(\frac{r_{1}^{2}}{J_{1} r_{2}}+\frac{r_{2}}{J_{2}}(1-\hat{\lambda})\right) \widehat{F}_{x} \\
& +\frac{1}{\widehat{x}_{2}}\left(\frac{r_{1}}{J_{1} r_{2}} M_{10}-\frac{1}{J_{2}} M_{20}(1-\widehat{\lambda})\right) \\
& +\left(\frac{d_{1}}{J_{1}}-\frac{d_{2}}{J_{2}}\right)(1-\hat{\lambda})-\frac{1}{\widehat{x}_{2}}\left(\frac{r_{1}}{r_{2}} k_{o 1}-(1-\hat{\lambda}) k_{o 2}\right) e_{1} \\
& -\dot{\lambda}_{\text {ref }}+\frac{r_{1}}{J_{1} r_{2}} \frac{1}{\widehat{x}_{2}} T_{b},
\end{aligned}
$$

where $\hat{\lambda}$ is given by (11) and $\lambda_{\text {ref }}$ is a desired slip signal to be imposed [33], having bounded derivative. The dynamic controller, defined for $\widehat{x}_{2} \neq 0$ (an appropriate saturation in (10) can avoid having $\widehat{x}_{2}$ approaching zero),

$$
\begin{aligned}
\dot{I}_{\widehat{e}}=\widehat{e}, & \\
T_{b}=\frac{J_{1} r_{2}}{r_{1}} \widehat{x}_{2}[- & k_{s 0} I_{\widehat{e}}-k_{s 1} \widehat{e}+\frac{1}{\widehat{x}_{2}}\left(\frac{r_{1}^{2}}{J_{1} r_{2}}+\frac{r_{2}}{J_{2}}(1-\hat{\lambda})\right) \\
& \cdot F_{x}(\widehat{\lambda})-\frac{1}{\widehat{x}_{2}}\left(\frac{r_{1}}{J_{1} r_{2}} M_{10}-\frac{1}{J_{2}} M_{20}(1-\hat{\lambda})\right) \\
& -\left(\frac{d_{1}}{J_{1}}-\frac{d_{2}}{J_{2}}\right)(1-\hat{\lambda}) \\
& \left.+\frac{1}{\widehat{x}_{2}}\left(\frac{r_{1}}{r_{2}} k_{o 1}-(1-\hat{\lambda}) k_{o 2}\right) e_{1}+\dot{\lambda}_{\mathrm{ref}}\right],
\end{aligned}
$$

with $k_{s 0}, k_{s 1}>0$, ensures that the tracking error $\hat{e}$ and its derivative globally and exponentially converge to zero, according to the relation

$$
\dot{\hat{e}}=-k_{s 1} \widehat{e}-k_{s 0} I_{\widehat{e}}
$$

or deriving

$$
\ddot{\hat{e}}+k_{s 1} \dot{\hat{e}}+k_{s 0} \widehat{e}=0 .
$$

Hence, $\hat{\lambda}$ tends to $\lambda_{\text {ref }}$ globally and exponentially. Since the observer ensures that $\widehat{\lambda} \rightarrow \lambda$ globally and exponentially, one concludes that also $\lambda$ tends to $\lambda_{\text {ref }}$ globally and exponentially.

It is worth noting that the dynamic controller (10), (24) requires that $M_{10}, M_{20}$ and all the system parameters are 
known. However, in practice, usually one has to deal with parameter uncertainties. In the remainder of this section we show that, in the case of bounded parameter uncertainties, the proposed controller still ensures global exponential stability, but to a proper ball of the origin. In fact, considering the estimated values $\widehat{J}_{1}, \widehat{J}_{2}, \widehat{d}_{1}, \widehat{d}_{2}, \widehat{r}_{1}, \widehat{r}_{2}, \widehat{M}_{10}$, and $\widehat{M}_{20}$, the observer (10)

$$
\begin{gathered}
\dot{\hat{x}}_{1}=\frac{\widehat{r}_{1}}{\widehat{J}_{1}} \widehat{F}_{x}-\frac{1}{\widehat{J}_{1}}\left(\widehat{d}_{1} \widehat{x}_{1}+\widehat{M}_{10}+T_{b}\right)+k_{o 1}\left(x_{1}-\widehat{x}_{1}\right), \\
\dot{\hat{x}}_{2}=-\frac{\widehat{r}_{2}}{\widehat{J}_{2}} \widehat{F}_{x}-\frac{1}{\widehat{J}_{2}}\left(\widehat{d}_{2} \widehat{x}_{2}+\widehat{M}_{20}\right)+k_{o 2}\left(x_{1}-\widehat{x}_{1}\right),
\end{gathered}
$$

and the control (24)

$$
\begin{aligned}
T_{b}=\frac{\widehat{J}_{1} \widehat{r}_{2}}{\widehat{r}_{1}} \widehat{x}_{2}[ & -k_{s 0} I_{\widehat{e}}-k_{s 1} \widehat{e}+\frac{1}{\widehat{x}_{2}}\left(\frac{\widehat{r}_{1}^{2}}{\widehat{J}_{1} \widehat{r}_{2}}+\frac{\widehat{r}_{2}}{\widehat{J}_{2}}(1-\widehat{\lambda})\right) \\
& \cdot F_{x}(\widehat{\lambda})-\frac{1}{\widehat{x}_{2}}\left(\frac{\widehat{r}_{1}}{\widehat{J}_{1} \widehat{r}_{2}} \widehat{M}_{10}-\frac{1}{\widehat{J}_{2}} \widehat{M}_{20}(1-\widehat{\lambda})\right) \\
& -\left(\frac{\widehat{d}_{1}}{\widehat{J}_{1}}-\frac{\widehat{d}_{2}}{\widehat{J}_{2}}\right)(1-\widehat{\lambda}) \\
& \left.+\frac{1}{\widehat{x}_{2}}\left(\frac{\widehat{r}_{1}}{\widehat{r}_{2}} k_{o 1}-(1-\widehat{\lambda}) k_{o 2}\right) e_{1}+\dot{\lambda}_{\text {ref }}\right],
\end{aligned}
$$

the observer error dynamics become

$$
\begin{aligned}
& \dot{e}_{1}=-\left(k_{o 1}+\frac{r_{1}}{J_{1}}\right) e_{1}+\frac{r_{1}}{J_{1}}\left(F_{x}-\widehat{F}_{x}\right)+\delta_{p 1}, \\
& \dot{e}_{2}=-k_{o 2} e_{1}-\frac{d_{2}}{J_{2}} e_{2}+\frac{r_{2}}{J_{2}}\left(F_{x}-\widehat{F}_{x}\right)+\delta_{p 2},
\end{aligned}
$$

while the tracking error dynamics become

$$
\dot{\hat{e}}=-k_{s 0} I_{\widehat{e}}-k_{s 1} \widehat{e}+\Delta_{p},
$$

where

$$
\begin{aligned}
\delta_{p 1}= & \left(\frac{r_{1}}{J_{1}}-\frac{\widehat{r}_{1}}{\widehat{J}_{1}}\right) \widehat{F}_{x}-\left(\frac{d_{1}}{J_{1}}-\frac{\widehat{d}_{1}}{\widehat{J}_{1}}\right) \widehat{x}_{1}-\frac{M_{10}}{J_{1}}+\frac{\widehat{M}_{10}}{\widehat{J}_{1}} \\
& -\left(\frac{1}{J_{1}}-\frac{1}{\widehat{J}_{1}}\right) \frac{\widehat{J}_{1} \widehat{r}_{2}}{\widehat{r}_{1}} \widehat{x}_{2}
\end{aligned}
$$

$$
\begin{aligned}
& \cdot\left[-k_{s 0} I_{\widehat{e}}-k_{s 1} \widehat{e}+\frac{1}{\widehat{x}_{2}}\left(\frac{\widehat{r}_{1}^{2}}{\widehat{J}_{1} \widehat{r}_{2}}+\frac{\widehat{r}_{2}}{\widehat{J}_{2}}(1-\widehat{\lambda})\right) F_{x}(\widehat{\lambda})\right. \\
& -\frac{1}{\widehat{x}_{2}}\left(\frac{\widehat{r}_{1}}{\widehat{J}_{1} \widehat{r}_{2}} \widehat{M}_{10}-\frac{1}{\widehat{J}_{2}} \widehat{M}_{20}(1-\hat{\lambda})\right) \\
& -\left(\frac{\widehat{d}_{1}}{\widehat{J}_{1}}-\frac{\widehat{d}_{2}}{\widehat{J}_{2}}\right)(1-\widehat{\lambda}) \\
& \left.+\frac{1}{\widehat{x}_{2}}\left(\frac{\widehat{r}_{1}}{\widehat{r}_{2}} k_{o 1}-(1-\widehat{\lambda}) k_{o 2}\right) e_{1}+\dot{\lambda}_{\text {ref }}\right] \text {, } \\
& \delta_{p 2}=-\left(\frac{r_{2}}{J_{2}}-\frac{\widehat{r}_{2}}{\widehat{J}_{2}}\right) \widehat{F}_{x}-\left(\frac{d_{2}}{J_{2}}-\frac{\widehat{d}_{2}}{\widehat{J}_{2}}\right) \widehat{x}_{2}-\frac{M_{20}}{J_{2}}+\frac{\widehat{M}_{20}}{\widehat{J}_{2}} \text {, } \\
& \Delta_{p}=-\frac{1}{\widehat{x}_{2}}\left(\frac{r_{1}^{2}}{J_{1} r_{2}}-\frac{\widehat{r}_{1}^{2}}{\widehat{J}_{1} \widehat{r}_{2}}+\left(\frac{r_{2}}{J_{2}}-\frac{\widehat{r}_{2}}{\widehat{J}_{2}}\right)(1-\widehat{\lambda})\right) \widehat{F}_{x} \\
& +\frac{1}{\widehat{x}_{2}}\left(\frac{r_{1}}{J_{1} r_{2}} M_{10}-\frac{\widehat{r}_{1}}{\widehat{J}_{1} \widehat{r}_{2}} \widehat{M}_{10}\right. \\
& \left.-\left(\frac{M_{20}}{J_{2}}-\frac{\widehat{M}_{20}}{\widehat{J}_{2}}\right)(1-\widehat{\lambda})\right) \\
& +\left(\frac{d_{1}}{J_{1}}-\frac{\widehat{d}_{1}}{\widehat{J}_{1}}-\frac{d_{2}}{J_{2}}+\frac{\widehat{d}_{2}}{\widehat{J}_{2}}\right)(1-\hat{\lambda}) \\
& -\frac{1}{x_{2}}\left(\frac{r_{1}}{r_{2}}-\frac{\widehat{r}_{1}}{\widehat{r}_{2}}\right) k_{o 1} e_{1}+\left(\frac{\widehat{J}_{1} r_{1} \widehat{r}_{2}}{J_{1} \widehat{r}_{1} r_{2}}-1\right) \\
& \cdot\left[-k_{s 0} I_{\widehat{e}}-k_{s 1} \widehat{e}+\frac{1}{\widehat{x}_{2}}\left(\frac{\widehat{r}_{1}^{2}}{\widehat{J}_{1} \widehat{r}_{2}}+\frac{\widehat{r}_{2}}{\widehat{J}_{2}}(1-\widehat{\lambda})\right) F_{x}(\widehat{\lambda})\right. \\
& -\frac{1}{\widehat{x}_{2}}\left(\frac{\widehat{r}_{1}}{\widehat{J}_{1} \widehat{r}_{2}} \widehat{M}_{10}-\frac{1}{\widehat{J}_{2}} \widehat{M}_{20}(1-\hat{\lambda})\right) \\
& -\left(\frac{\widehat{d}_{1}}{\widehat{J}_{1}}-\frac{\widehat{d}_{2}}{\widehat{J}_{2}}\right)(1-\widehat{\lambda}) \\
& \left.+\frac{1}{\widehat{x}_{2}}\left(\frac{\widehat{r}_{1}}{\widehat{r}_{2}} k_{o 1}-(1-\widehat{\lambda}) k_{o 2}\right) e_{1}+\dot{\lambda}_{\text {ref }}\right]
\end{aligned}
$$

are the perturbation terms. Deriving the closed loop dynamics, one gets

$$
\ddot{\widetilde{e}}+k_{s 1} \dot{\hat{e}}+k_{s 0} \widehat{e}=\Psi_{p},
$$

where $\Psi_{p}=\dot{\Delta}_{p}$. If $\left|\delta_{1}\right|,\left|\delta_{2}\right| \leq \delta_{\max },\left|\Psi_{p}\right| \leq \Psi_{\max }$ remain bounded; then the errors $e_{1}, e_{2}$, and $\widehat{e}$ will be uniformly ultimately bounded [25]. In fact, since

$$
\left(\begin{array}{c}
\dot{\hat{e}} \\
\ddot{\vec{e}}
\end{array}\right)=A\left(\begin{array}{c}
\widehat{e} \\
\dot{e}
\end{array}\right)+B \Psi_{p}, \quad A=\left(\begin{array}{cc}
0 & 1 \\
-k_{s 0} & -k_{s 1}
\end{array}\right), B=\left(\begin{array}{l}
0 \\
1
\end{array}\right),
$$


and considering the Lyapunov candidate $V=V_{o}+V_{s}, V_{s}=$ $\left(\begin{array}{c}\widehat{e} \\ \grave{\hat{e}}\end{array}\right)^{T} P\left(\begin{array}{c}\widehat{e} \\ \dot{e}\end{array}\right)$, with $P=P^{T}>0$ solution of Sylvester's equation $P A+A^{T} P=-Q$, for a fixed matrix $Q=Q^{T}>0$, one works out

$$
\begin{aligned}
& \dot{V} \leq-\left(1-\vartheta_{1}\right) \lambda\left\|\begin{array}{l}
e_{1} \\
e_{2}
\end{array}\right\|^{2}-\left(1-\vartheta_{2}\right) \lambda_{\min }^{\mathrm{Q}} \| \begin{array}{l}
\hat{e} \\
\dot{\hat{e}} \|^{2}
\end{array} \\
& +\left\|\begin{array}{l}
e_{1} \\
e_{2}
\end{array}\right\|\left(\delta_{\max }-\vartheta_{1} \lambda\left\|\begin{array}{l}
e_{1} \\
e_{2}
\end{array}\right\|\right) \\
& +\left\|\begin{array}{c}
\hat{e} \\
\dot{\hat{e}}
\end{array}\right\|\left(\|P B\| \Psi_{p}-\vartheta_{2} \lambda_{\min }^{Q} \| \begin{array}{l}
\hat{e} \\
\dot{\hat{e}}
\end{array}\right) \\
& \leq-\left(1-\vartheta_{1}\right) \lambda\left\|\begin{array}{l}
e_{1} \\
e_{2}
\end{array}\right\|^{2}-\left(1-\vartheta_{2}\right) \lambda_{\min }^{\mathrm{Q}} \| \begin{array}{l}
\hat{e} \\
\dot{\hat{e}} \|^{2}
\end{array}
\end{aligned}
$$

with $\vartheta_{1}, \vartheta_{2} \in(0,1)$ and $\lambda_{\min }^{Q}$ being the minimum eigenvalue of $Q$, for

$$
\left\|\begin{array}{l}
e_{1} \\
e_{2}
\end{array}\right\| \geq \frac{1}{\vartheta_{1} \lambda} \delta_{\max }, \quad\|\hat{e}\| \geq \frac{\|P B\|}{\vartheta_{2} \lambda{ }_{\min }^{Q}} \Psi_{\max } .
$$

This implies that $\left(e_{1}, e_{2}\right)$ and $(\hat{e}, \dot{\hat{e}})$ will be globally ultimately bounded to balls of radius

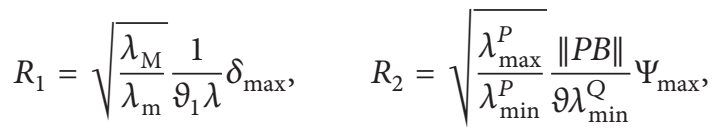

of the origin, respectively, with $\lambda_{\mathrm{m}}=\min \{1, \gamma\}, \lambda_{\mathrm{M}}=$ $\max \{1, \gamma\}$, and $\lambda_{\min }^{P}, \lambda_{\max }^{P}$ being the minimum and maximum eigenvalues of $P$.

\section{Experimental Results}

Experimental tests on Inteco's ABS Laboratory setup (see Figure 4) have been conducted to evaluate the braking performance of the controller (10), (24). These tests represent maneuvers in straight line. Moreover, in order to compare the performance of the proposed controller with another available in literature, a comparison with a sliding-mode controller proposed in [16] is presented.

The initial value of the angular velocities of the upper and lower wheels is $158 \mathrm{rad} / \mathrm{s}$. When the maximal velocity of the upper wheel is detected, the system disables the DC motor coupled to the lower wheel, and the braking process begins.

The observer initial conditions for the proposed controller are $150 \mathrm{rad} / \mathrm{s}$, for the upper and lower wheels. The observer initial conditions in the sliding-mode controller are obviously the same. The observer gain $k_{o 2}$ can be determined as indicated in the previous section. Considering $\bar{x}_{2} \in$ $[-170,170]$ and $\bar{\lambda} \in[0,1]$, one determines $c_{1}^{\circ}=2, c_{2}^{\circ}=$ 10 , and $k_{o 2}=40$. It is worth noting that in this work we have considered $\mu_{x}=1$, but its variability can be taken into account considering the maximization procedure used to determine $c_{1}^{\circ}, c_{2}^{\circ}$, making varying $\mu_{x}$ in the interval of interest.

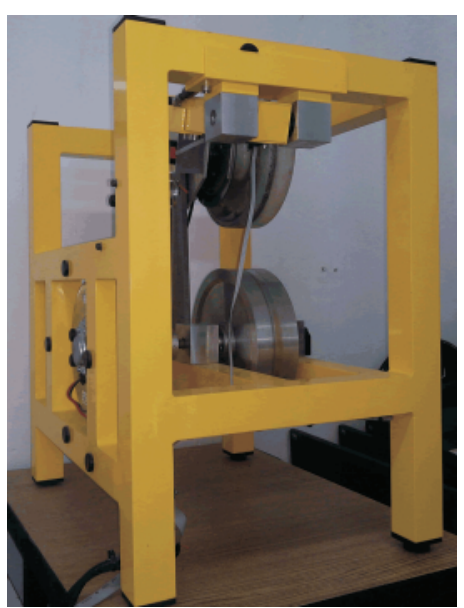

Figure 4: The ABS Laboratory setup.

The results are summarized in Figures 5-9, where it can be seen that the proposed controller (10), (24) ensures better performances with respect to the sliding-mode controller [16]. It is worth noting that, after the braking phase, between 5.5 and $7 \mathrm{~s}$, corresponding to the maximum braking efficiency, the performance after $7 \mathrm{~s}$ is no longer relevant since the velocity is low, and the ABS is not working anymore in the appropriate range of velocities.

Comparing Figures 5 and 6, one can see that the controller (10), (24) maintains a lower estimation error than the observer with the sliding-mode control. Furthermore, as shown in Figure 7, the proposed controller reaches the reference $\lambda_{\text {ref }}=0.15$ faster and keeps the estimated slip closer to the reference, with respect to the sliding-mode control. As already mentioned, at the end of the braking process the reference tracking is meaningless, since the longitudinal velocity is low and the ABS system is no longer effective. Another positive effect of the proposed controller is shown in Figure 8, where the absence of chattering, typical of the sliding-mode control, can be appreciated, thus ensuring a better wear resistance, less noise, and an increased passenger comfort with lower jerk effects. As a final indication of the better performance of the proposed controller, Figure 9 shows that the controller (10), (24) ensures (even if marginally) shorter braking spaces.

\section{Conclusions}

This paper presents a dynamic controller for an ABS Laboratory setup, based on a state observer of the angular velocities of the two wheels constituting the setup. The acceleration and velocity of the upper wheel, which simulates the automobile wheel, are measurable. This is a reasonable assumption in modern vehicles equipped with an ABS system. The bearing friction torques and the system parameters are assumed to be known. The nonlinear observer is designed considering a simplified Pacejka tire model for the upper wheel. The nonlinear dynamic controller is finally designed, ensuring the exponential stability of the system. 


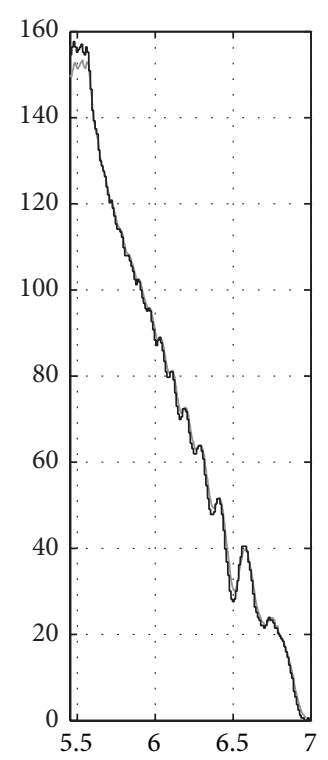

(a)

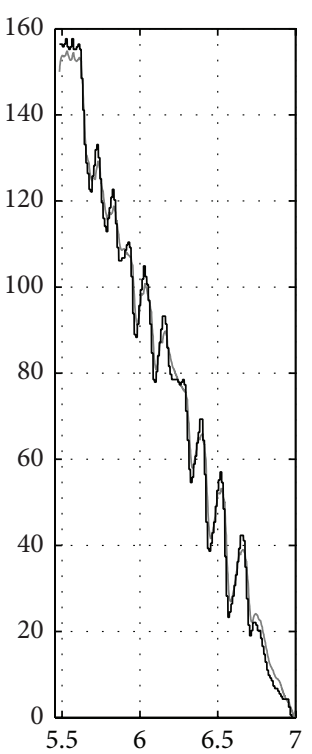

(b)

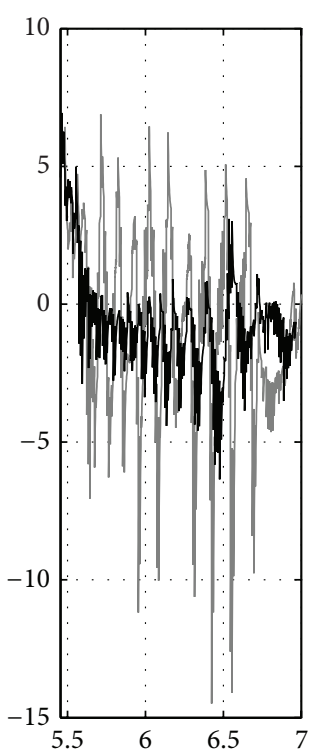

(c)

FIGURE 5: (a) Controller (10), (24): upper wheel angular velocity $x_{1}$ (black) and estimation $\widehat{x}_{1}$ (gray) (rad/s versus s); (b) sliding-mode control: upper wheel angular velocity $x_{1}$ (black) and estimation $\widehat{x}_{1}$ (gray) (rad/s versus s); (c) estimation error $x_{1}-\widehat{x}_{1}$ : controller (10), (24) (black) and sliding-mode control (gray) ( $\mathrm{rad} / \mathrm{s}$ versus s).

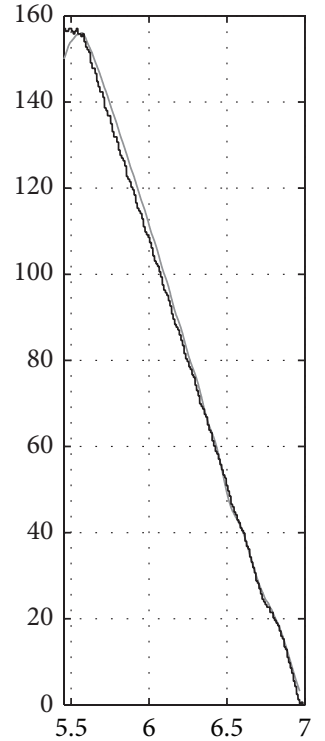

(a)

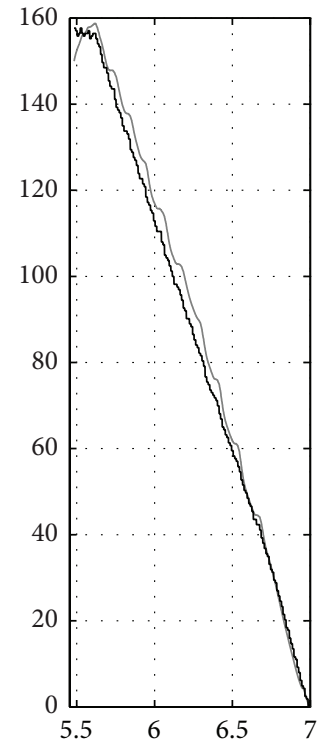

(b)

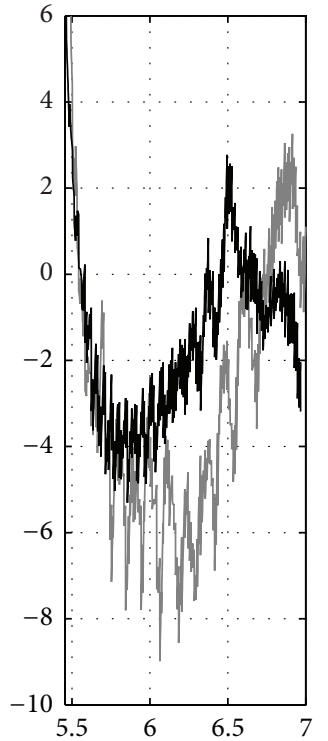

(c)

Figure 6: (a) Controller (10), (24): lower wheel angular velocity $x_{2}$ (black) and estimation $\widehat{x}_{2}$ (gray) (rad/s versus s); (b) sliding-mode control: lower wheel angular velocity $x_{2}$ (black) and estimation $\widehat{x}_{2}$ (gray) (rad/s versus s); (c) estimated error $x_{2}-\widehat{x}_{2}$ : controller (10), (24) (black) and sliding-mode control (gray) ( $\mathrm{rad} / \mathrm{s}$ versus s).

A series of experiments have been performed on the ABS Laboratory setup to check the performance of the proposed dynamic controller, for different cases and conditions. The experimental results also show the performance of this dynamic controller in comparison with a slidingmode control proposed in the literature. This latter suffers from chattering, which on the contrary is absent in the proposed controller, which hence shows better performances. Moreover, the proposed controller achieves shorter braking spaces in shorter times, thus increasing the safety. Finally, thanks to smoother signal to the actuator with respect to the sliding-mode control, the proposed controller ensures longer durability of the pads, less noise, and an increased passenger comfort with lower jerk effects. 


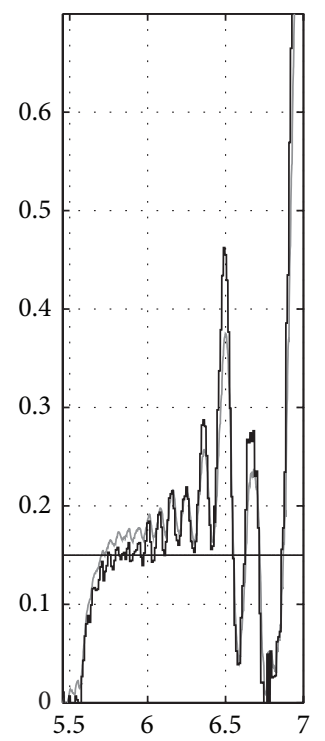

(a)

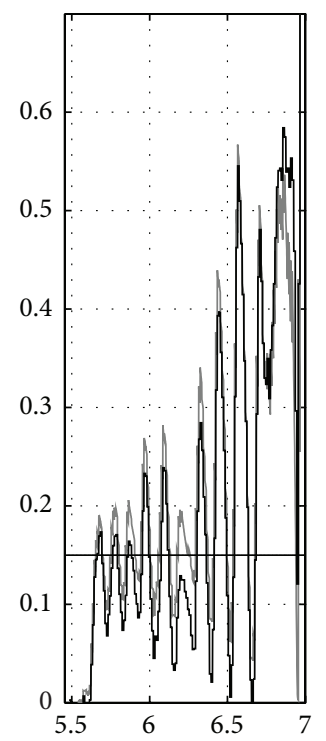

(b)

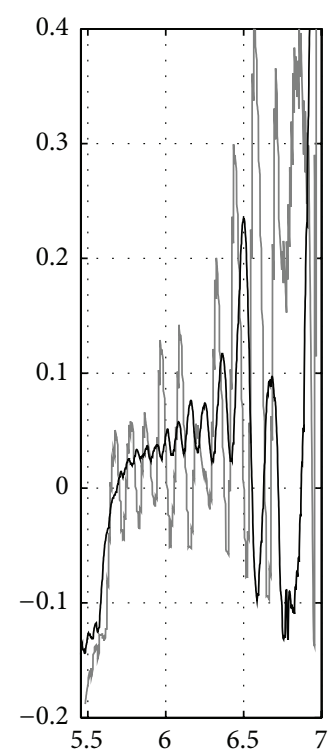

(c)

Figure 7: (a) Controller (10), (24): wheel slip $\lambda$ (solid black), estimated wheel slip $\hat{\lambda}$ (solid gray), and wheel slip reference $\hat{\lambda}_{\text {ref }}$ (dashed) (dimensionless versus s); (b) sliding-mode control: wheel slip $\lambda$ (solid black), estimated wheel slip $\hat{\lambda}$ (solid gray), and wheel slip reference $\widehat{\lambda}_{\text {ref }}$ (dashed) (dimensionless versus s); (c) tracking error $\widehat{\lambda}-\lambda_{\text {ref: }}$ controller (10), (24) (black) and sliding-mode control (gray) (dimensionless versus s).

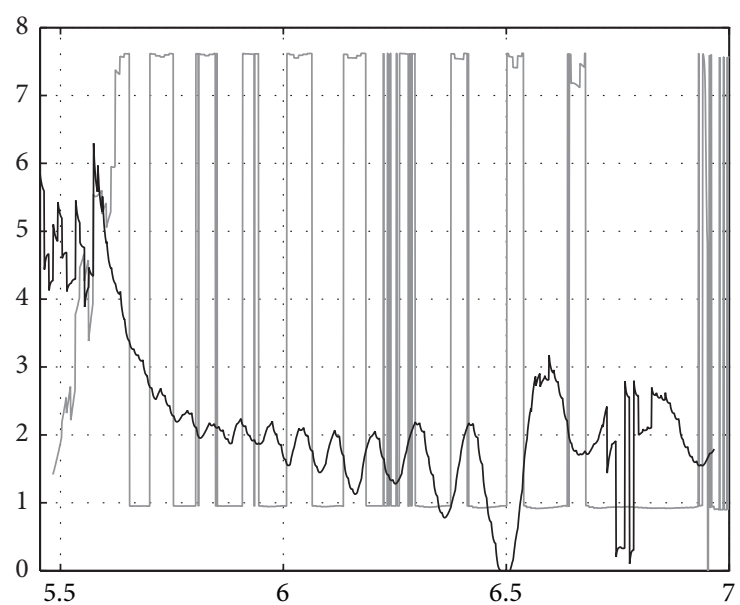

FIgURE 8: Input control applied to system $T_{b}$ ( $\mathrm{N}$ m versus s): controller (10), (24) (black) and sliding-mode control (gray).

\section{Conflict of Interests}

The authors declare that there is no conflict of interests regarding the publication of this paper.

\section{Acknowledgment}

The authors would like to thank Iordan Javier Mares Gúzman for his help in the realization of the experimental results, carried out during the realization of his M.S. thesis at the Universidad de Guadalajara.

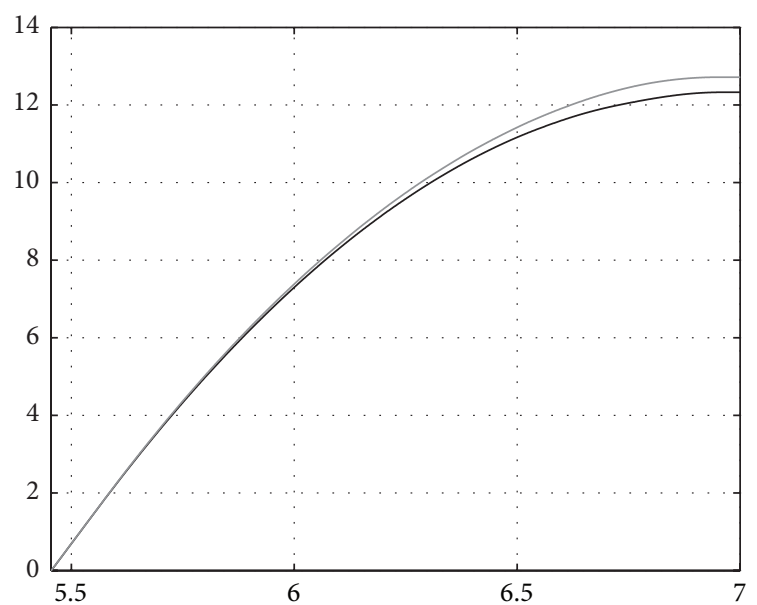

FIGURE 9: Braking distance (m versus s): controller (10), (24) (black) and sliding-mode control (gray).

\section{References}

[1] M. Petrov, V. Balankin, and O. Naruzhnyi, "Study of automobiles brakes and pneumatic tires work model of the work process of antiblock brake system," Tech. Rep., NISI, Novosibirsk, Russia, 1977.

[2] N. Rittmannsberger, "Antilock braking system and traction control," in Proceedings of the International Congress on Transportation Electronics Convergence, pp. 195-202, 1988.

[3] R. Emig, H. Goebels, and J. Schramm, "Antilock braking systems (ABS) for commercial vehicles-status 1990 and future prospects," in Proceedings of the International Congress on 
Transportation Electronics, 1990. Vehicle Electronics in the 90's, pp. 515-523, 1990.

[4] U. Kiencke and L. Nielsen, Automotive Control Systems: For Engine, Driveline, and Vehicle, Springer, 2nd edition, 2010.

[5] R. Rajamani, Vehicle Dynamics and Control, Springer, New York, NY, USA, 2006.

[6] Robert Bosch GmbH, "ABS-a success story," 2003, http://www .bosch.com/assets/en/company/innovation/theme03.htm.

[7] A. G. Daimler, "Mercedes-Benz and the invention of the antilock braking system: ABS, ready for production in 1978," 2008.

[8] Robert Bosch GmbH, Bosch Automotive Handbook, Bentley Publisher, 8th edition, 2011.

[9] E. Limberg and J. Enterprises, Introduction to Foundation Brake Design, SAE International, Ebook, 2014.

[10] Inteco User's Manual, The Laboratory Antilock Braking System Controlled from PC, Inteco Ltd., Crakow, Poland, 2006.

[11] M. H. Al-Mola, M. Mailah, P. M. Samin, A. H. Muhaimin, and M. Y. Abdullah, "Performance comparison between sliding mode control and active force control for a nonlinear anti-lock brake system," WSEAS Transactions on Systems and Control, vol. 9, pp. 101-107, 2014.

[12] S. John and J. O. Pedro, "A comparative study of two control schemes for anti-lock braking systems," in Proceedings of the Control Conference (ASCC '09), Istambul, Turkey, June 2009.

[13] M.-B. Radac, R.-E. Precup, S. Preitl, J. K. Tar, J. Fodor, and E. M. Petriu, "Gain-scheduling and iterative feedback tuning of PI controllers for longitudinal slip control," in Proceedings of the IEEE 6th International Conference on Computational Cybernetics (ICCC '08), pp. 183-188, November 2008.

[14] Y. Oniz, E. Kayacan, and O. Kaynak, "A dynamic method to forecast the wheel slip for antilock braking system and its experimental evaluation," IEEE Transactions on Systems, Man, and Cybernetics, Part B: Cybernetics, vol. 39, no. 2, pp. 551-560, 2009.

[15] L. Li, F.-Y. Wang, and Q. Zhou, "Integrated longitudinal and lateral tire/road friction modeling and monitoring for vehicle motion control," IEEE Transactions on Intelligent Transportation Systems, vol. 7, no. 1, pp. 1-19, 2006.

[16] E. Kayacan, Y. Oniz, and O. Kaynak, "A grey system modeling approach for sliding-mode control of antilock braking system," IEEE Transactions on Industrial Electronics, vol. 56, no. 8, pp. 3244-3252, 2009.

[17] R.-E. Precup, S. V. Spătaru, M.-B. Radac, E. M. Petriu, S. Preitl, and C.-A. Dragoş, "Model-based fuzzy control solutions for a laboratory Antilock Braking System," in Proceedings of the 3rd International Conference on Human System Interaction (HSI '10), pp. 133-138, May 2010.

[18] R. E. Precup, S. V. Spătaru, M. B. Rădac et al., "Experimental results of model-based fuzzy control solutions for a laboratory antilock braking system," in Human-Computer Systems Interaction: Backgrounds and Applications 2, vol. 99 of Advances in Intelligent and Soft Computing, pp. 223-234, Springer, Berlin, Germany, 2012.

[19] M. B. Radac, R. E. Precup, S. Preitl, J. K. Tar, and E. M. Petriu, "Linear and fuzzy control solutions for a laboratory antilock braking system," in Proceedings of the 6th International Symposium on Intelligent Systems and Informatics (SISY '08), pp. $1-6,2008$.

[20] M. Habibi and A. Yazdizadeh, "A new fuzzy logic road detector for antilock braking system application," in Proceedings of the 8th IEEE International Conference on Control and Automation (ICCA '10), pp. 1036-1041, IEEE, Xiamen, China, June 2010.
[21] M. A. Khanesar, E. Kayacan, M. Teshnehlab, and O. Kaynak, "Extended kalman filter based learning algorithm for type2 fuzzy logic systems and its experimental evaluation," IEEE Transactions on Industrial Electronics, vol. 59, no. 11, pp. 44434455, 2012.

[22] A. V. Topalov, E. Kayacan, Y. Oniz, and O. Kaynak, "Adaptive neuro-fuzzy control with sliding mode learning algorithm: application to antilock braking system," in Proceedings of the 7th Asian Control Conference (ASCC '09), Hong Kong, China, August 2009.

[23] A. V. Topalov, E. Kayacan, Y. Oniz, and O. Kaynak, "Neurofuzzy control of antilock braking system using variablestructure- systems-based learning algorithm," in Proceedings of the International Conference on Adaptive and Intelligent Systems (ICAIS '09), pp. 166-171, September 2009.

[24] A. V. Topalov, Y. Oniz, E. Kayacan, and O. Kaynak, "Neurofuzzy control of antilock braking system using sliding mode incremental learning algorithm," Neurocomputing, vol. 74, no. 11, pp. 1883-1893, 2011.

[25] H. K. Khalil, Nonlinear Systems, Prentice Hall, 3rd edition, 2002.

[26] G.-M. Chen, W.-Y. Wang, T.-T. Lee, and C. W. Tao, “Observerbased direct adaptive fuzzy-neural control for anti-lock braking systems," International Journal of Fuzzy Systems, vol. 8, no. 4, pp. 208-218, 2006.

[27] L.-H. Zhao, Z.-Y. Liu, and H. Chen, "Design of a nonlinear observer for vehicle velocity estimation and experiments," IEEE Transactions on Control Systems Technology, vol. 19, no. 3, pp. 664-672, 2011.

[28] L. Imsland, H. F. Grip, T. A. Johansen, T. I. Fossen, J. C. Kalkkuhi, and A. Suissa, "Nonlinear observer for vehicle velocity with friction and road bank angle adaptation-validation and comparison with an extended kalman filter," in Proceedings of the SAE World Congress and Exhibition, 2007.

[29] G. Baffet, J. Stephant, and A. Charara, "Lateral vehicle dynamic observers: simulations and experiments," International Journal of Vehicle Autonomous Systems, vol. 5, no. 4, pp. 184-203, 2007.

[30] J. Stéphant, A. Charara, and D. Meizel, "Evaluation of a sliding mode observer for vehicle sideslip angle," Control Engineering Practice, vol. 15, no. 7, pp. 803-812, 2007.

[31] H. B. Pacejka, Tyre and Vehicle Dynamics, Elsevier ButterworthHein, New York, NY, USA, 2006.

[32] A. Dadashnialehi, A. Bab-Hadiashar, Z. Cao, and A. Kapoor, "Accurate wheel speed measurement for sensorless ABS in electric vehicle," in Proceedings of the IEEE International Conference on Vehicular Electronics and Safety (ICVES '12), pp. 37-42, Istanbul, Turkey, July 2012.

[33] A. Zanten, R. Erhardt, and A. Lutz, "Measurement and simulation of transient in longitudinal and lateral tire forces," Society of Automotive Engineers, vol. 99, no. 6, pp. 300-318, 1990. 


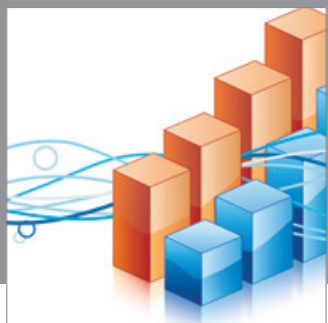

Advances in

Operations Research

mansans

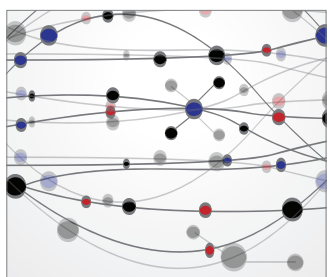

The Scientific World Journal
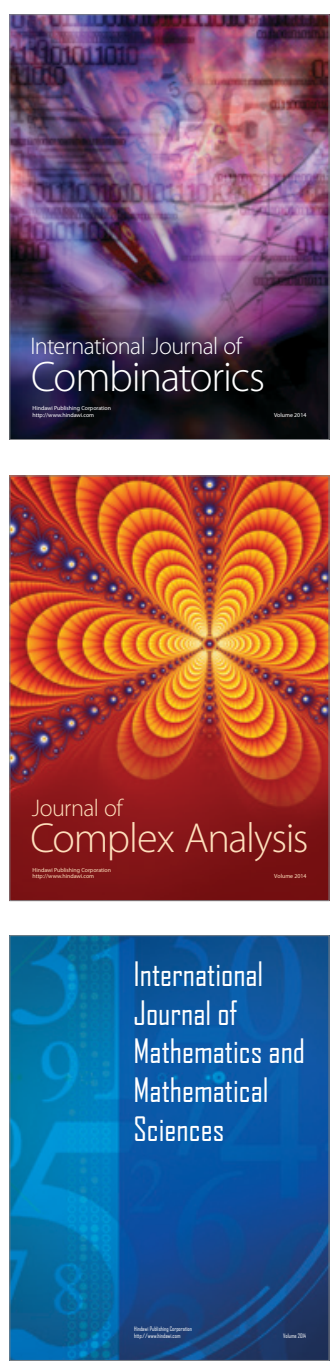
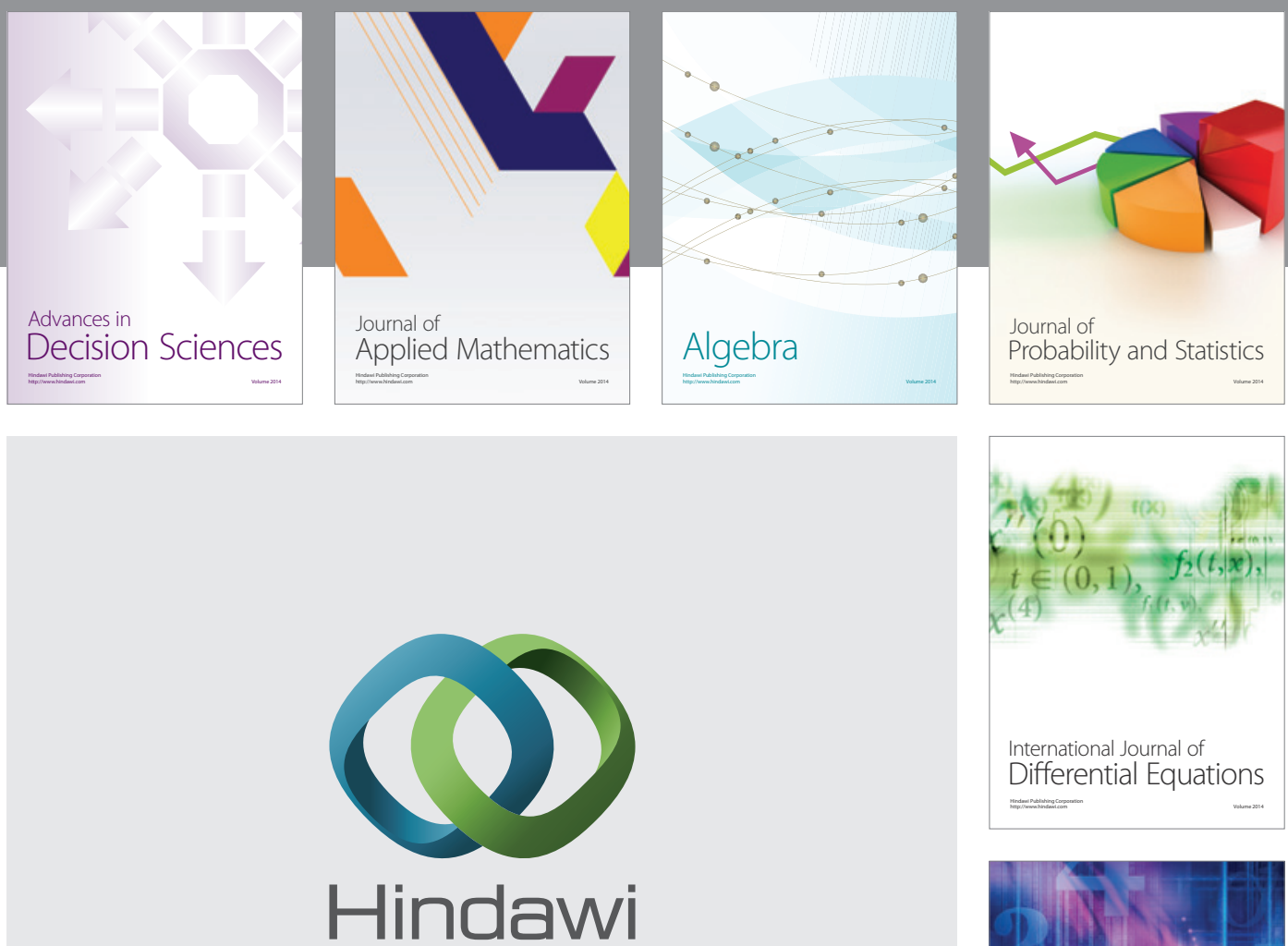

Submit your manuscripts at http://www.hindawi.com
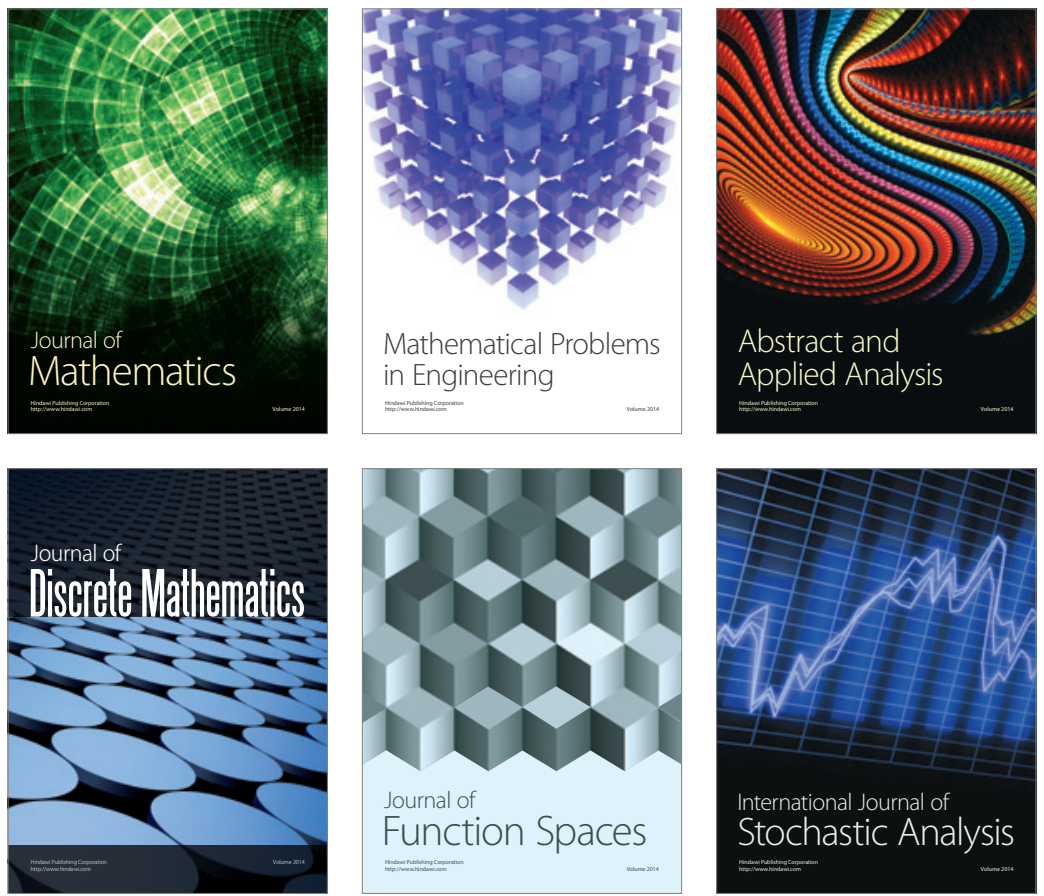

Journal of

Function Spaces

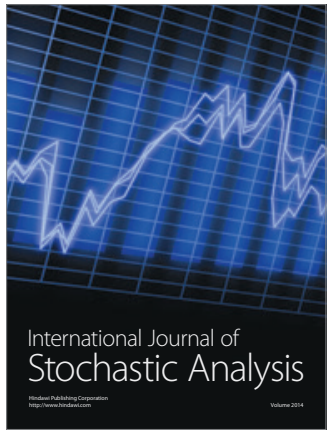

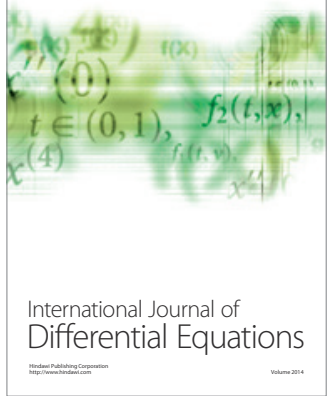
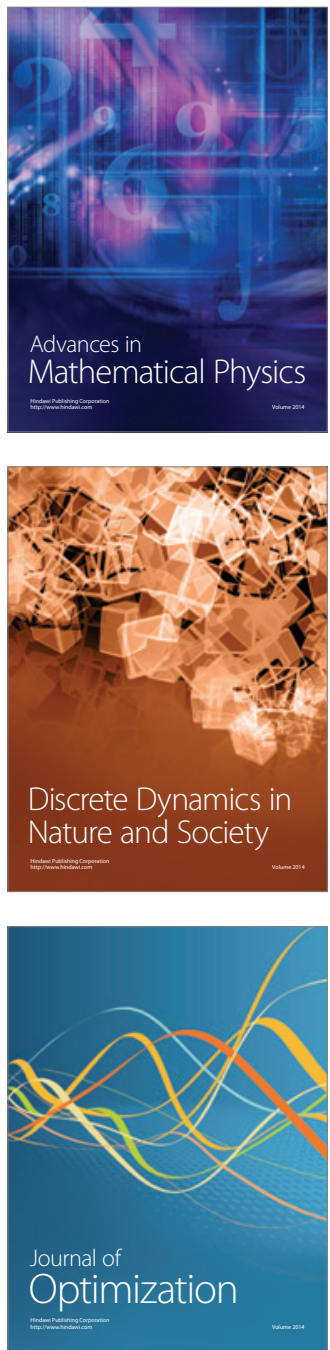